\title{
Strong Currents Don't Faze Salt Marsh Coastal Defenses
}

\section{A model captures the influence of plant flexibility, leaves, and current on wave dissipation by a meadow of marsh plants.}

\author{
By Rachel Berkowitz
}

$\mathrm{M}$ arsh grasses that carpet tidal flats provide vital habitats for diverse flora and fauna, and by dissipating wave energy, they also defend against coastal storms. Facilitating policies to restore and manage these fragile environments requires predictions of how plants interact with waves and currents to dissipate hydrodynamic energy. Xiaoxia Zhang at Dalian University of Technology in China and Heidi Nepf at the Massachusetts Institute of Technology now provide those predictions with a model that captures the dynamics of plant components bending in response to fluid motion [1].

Many wave-dissipation models treat vegetation as rigid cylinders with empirically determined drag coefficients. Those models cannot be applied to all plants and environments because drag depends on plant morphology, mechanical properties, and local currents. To account for these parameters, Zhang and Nepf modeled the forces applied to individual plants by back-and-forth wave motion and sustained onshore or offshore currents. They found that, for all plant types, wave-energy dissipation was greatest when current velocity was several multiples of wave velocity. For realistic (flexible) plants, less wave energy was dissipated overall compared with rigid plants, and the degree of dissipation was less sensitive to the ratio of current velocity to wave velocity. That's because the motion of flexible stems, and drag from leaves, reduced both wave and current velocities, mitigating the impact of currents on energy dissipation.

The researchers used their individual-plant model to determine Credit: X. Zhang/Dalian University of Technology

the forces on a tidal marsh and predict its wave-energy dissipation. They then confirmed this prediction by measuring wave decay in a physical model of flexible plants. Such predictions could help researchers and policy makers to estimate the value of marsh coastal defense as climate change brings stronger and more frequent coastal storms.

Rachel Berkowitz is a Corresponding Editor for Physics based in Vancouver, Canada.

\section{REFERENCES}

1. X. Zhang and H. Nepf, "Wave damping by flexible marsh plants influenced by current," Phys. Rev. Fluids 6, 100502 (2021). 